\title{
A Clinical Study on Juheli (Recombinant Human Interleukin - 11) in the Second Prevention of Chemotherapy Induced Thrombocytopenia
}

\author{
Yang Xiao ${ }^{1 \&}$, Jun Liu ${ }^{1 \&}$, Xin-En Huang²*, Jian-Xiong Guo, Peng-Chao Fu ${ }^{1}$, \\ Xiao-Hong Huang', Juan Zhou' ${ }^{1}$, Ai-Qin Ye ${ }^{1}$
}

\begin{abstract}
Objective: to investigate the effect and side effects of recombinant human interleukin - 11 (rhIL - 11, in Chinese Juheli, produced by Qi Lu Biotechnology CO., LTD) in the second prevention of chemotherapy induced thrombocytopenia (CIT). Methods: Cancer patients with CIT were recruited and were treated with rhIL 11 (treatment phase, TP), and in the following cycle, all these patients administered with rhIL - 1124 hours immediately after chemotherapy (preventive treatment phase,PTP). Duration and severity of thrombocytopenia between two phases were compared. Results: for patients in TP or PTP, nadir values of platelet were $(29.28 \pm 20.08) \times 10^{9} / \mathrm{L}$ and $(45.24 \pm 19.66) \times 10^{9} / \mathrm{L}$, duration of thrombocytopenia in TP and PTP was $(11.52 \pm 4.33)$ and $(8.20 \pm+2.77)$ days, recovery time was $(19.40 \pm 3.89)$ and $(13.44 \pm 3.02)$ days, duration of rhIL - 11 administration was $10.68 \pm 2.46)$ and $(6.28 \pm 1.77)$ days, number of patients needing platelet infusion was 16 and 4 respectively, all differences were statistically significant $(p$ value were $0.007,0.002,0.000,0.000,0.034$ respectively). For TP and PTP, number of patients with hemorrhage was 8 and 4, duration of bleeding was $(5.00 \pm 0.82)$ and $(4.50 \pm 0.71)$ days respectively, with no statistically significant difference. Adverse reactions mainly included fever, edema, arrhythmia, joint pain, fatigue, skin rash, headache, dizziness, etc., all were not statistically significant between TPand PTP. Conclusion: rhIL - 11 could be well tolerated and is effective that could reduce the duration, severity of CIT, platelet transfusion, and incidence of bleeding, as well as shorten the recovery time, duration of rhIL - 11 administration. Thus, rhIL - 11 could be commended in the second prevention of CIT for patients with cancer.
\end{abstract}

Keywords: Recombinant human interleukin - 11 - second prevention - chemotherapy induced thrombocytopenia

Asian Pac J Cancer Prev, 17 (2), 485-489

\section{Introduction}

Cancer and its treatment, especitally myelosuppressive chemotherapy are frequently accompanied by anemia, neutropenia, and thrombocytopenia, as well as some combination of these conditions. These complications are associated with the potential to produce life threatening neutropenia, anemia, and thrombocytopenia, and could potentially increase the morbidity. These conditions also could compromise therapeutic dosing of chemotherapy which impacts survival as well as quality of life. The introduction of recombinant growth factors could minimize or prevent the development of treatmentinduced anemia and neutropenia, but the management of chemotherapy-induced thrombocytopenia (CIT) remains a major challenge. Several guidelines recommend dose reduction in case of CIT, although full dose, on time chemotherapy could lead to reduced tumor burden and better overall survival (1a-3a). Thus, patients with CIT will experience potentially life threatening complications, delay in treatment, poorer outcomes and increases of health care resources (Elting et al., 2001). At present, platelet transfusions remain the "gold-standard" for the management of severe CIT, but there are many issues and possible complications associated with platelet transfusions (Clarke et al., 1996). Consequently, medical oncologists are engaged in development of an urgent method that could ameliorate CIT.

Recombinant human interleukin-11 (rHuIL-11) was the first thrombocytopoietic cytokine (Demetri et al., 2000), a protein product of bone marrow stromal cells (Teramura et al., 1996). RHuIL-11 could enhance the growth of early hematopoietic progenitor cells, and stimulate both megakaryocytopoiesis and erythropoiesis. Administration of recombinant IL-11 to myelosuppressed animals accelerates the recovery of multilineage blood 
Table 1. Patient Characteristics

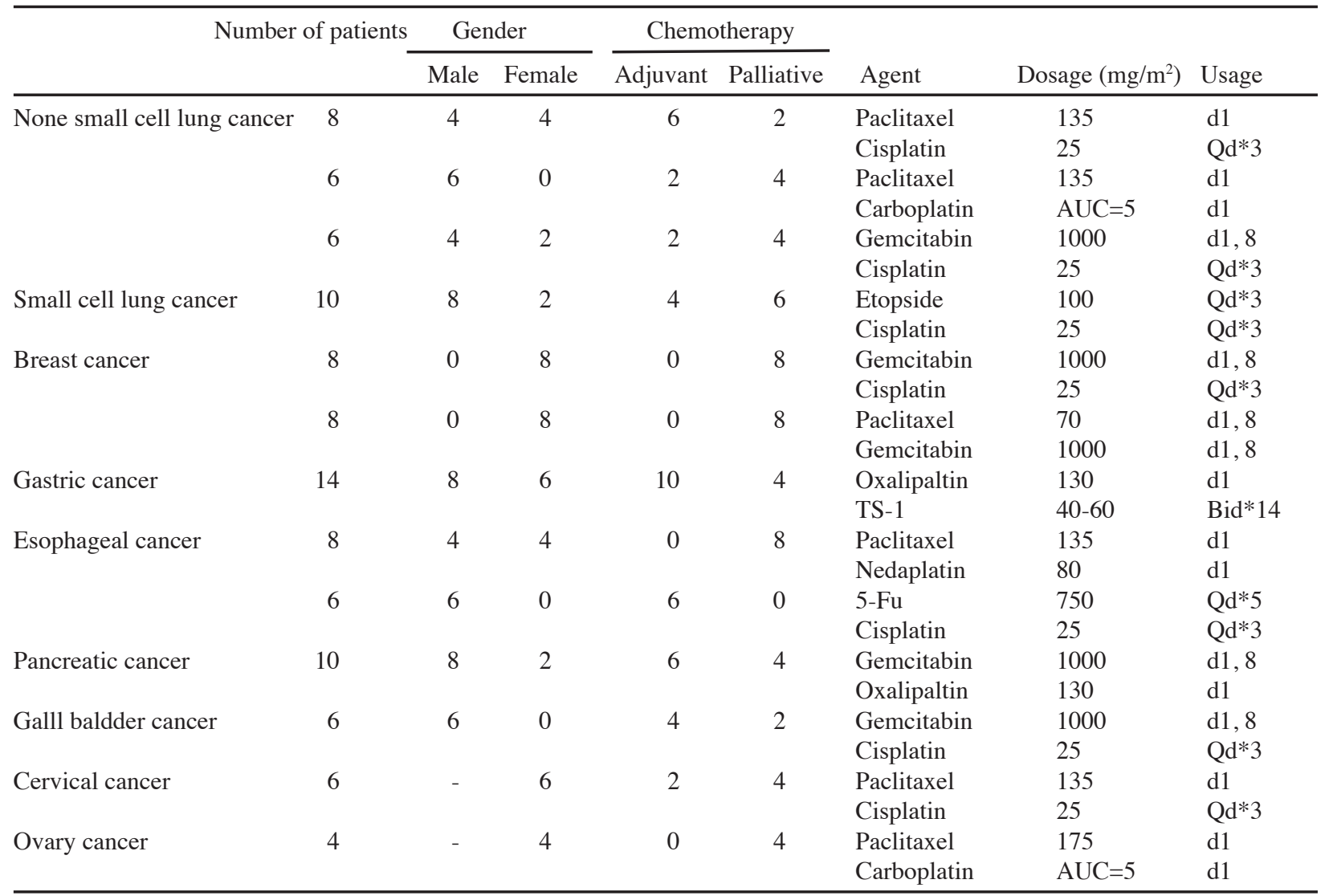

elements, including platelets.

Thus, it is licensed for the prevention of severe CIT and the reduction of the need for platelet transfusion following myelosuppressive chemotherapy in cancer patients who are at high risk of severe CIT. However, rHuIL-11 has significant side effects and demonstrates only modest efficacy. Therefore, optimizing the management of CIT remains a significant unmet need for patients with this condition (Demetri et al., 2000). And, few study is conducted to confirm the effectiveness of rhIL - 11 in the second prevention of CIT.

On this background, it is hypothesized that rhIL - 11 could be effective in the second prevention of CIT.

\section{Materials and Methods}

Patients recruited in this study were required to be pathologically/ cytologically diagnosed with cancer in Tai Xing Hospital from June 2015 to April 2013, to sign an informed consent before treatment; to expose to long term chemotherapy and supportive care; to have a score of Karnofsky Performance Status (KPS) $\geq 60$ with expectancy life span more than 3 moths; to be classified with no contraindications for chemotherapy; to have a routine blood test and confirmed thrombocytopenia (blood platelet less than $75 * 10^{9} /$ L) after chemotherapy, and normal hematopoietic function as evidenced by white blood cell count 3000/ul, normal hepatic function test (aspartate aminotransaminase and alanine aminotransferase less than 1.5 times of the upper limit of normal values), renal function test (serum total bilirubin $<1.5 \mathrm{mg} / \mathrm{dl}$ and creatinine $<1.5 \mathrm{mg} / \mathrm{dl}$ ). Exclusion criteria included history of alcoholic intoxication, diabetes, and patients who were pregnant or nursing. Chemotherapy was administered according to NCCN guideline. All patients were injected subcutaneously with recombinant human interleukin - 11 (rhIL - 11, in Chinese Juheli, produced by Qi Lu Biotechnology CO., LTD) at a dosage of $50 \mathrm{ug} / \mathrm{kg} / \mathrm{day}$, after the first cycle of chemotherapy when throbocytopenia was confirmed till the blood platelet count no less than 100 $* 10^{9} / \mathrm{L}$ (treatment phase, TP), and 24 hours immediately after thefollowing cycle, all these patients administered with rhIL - 11 till the blood platelet count no less than $100 * 10^{9} / \mathrm{L}$ (preventive treatment phase, PTP). After TP and PTP were attempted, safety and effectiveness were evaluated. We have enough experience in conducting medical researches, and have published some cancer related results elsewhere (Chen et al., 2014; Chen et al., 2014; Cao et al., 2014; Cui et al., 2014; Huang et al., 2014; Huang et al., 2014; Ji et al., 2014; Liu et al., 2014; Liu et al., 2014; Lu et al., 2014; Qian et al., Tian et al., 2014; 2014; Xiao et al., 2014; Xiao et al., 2014; Xu et al., 2014; $\mathrm{Xu}$ et al., 2014; Xu et al., 2014; Xu et al., 2014; Wang et al., 2014; Wu et al., 2014; Cui et al., 2015; Huang et al., 2015; Huang et al., 2015; Li et al., 2015; Liu et al., 2015; Liu et al., 2015; Qian et al., 2015; Shen et al., 2015; Shi et al., 2015; Sun et al., 2015; Xu et al., 2015; Xu et al., 2015; Xu et al., 2015; Xu et al., 2015; Wang et al., 2015; Wu et al., 2015; Wu et al., 2015; Yang et al., 2015; Zhou et al., 2015)

\section{Toxicity Evaluation}

The incidence rates of toxicity in this study were assessed on baseline and respectively after TP and PTP, 
Table 2. Difference between TP and PTP

\begin{tabular}{lcccr}
\hline & TP & PTP & $\mathrm{t} / \mathrm{x}^{2}$ & $p$ \\
\hline Nadir of platelet $\left(10^{9} / \mathrm{L}\right)$ & $29.28 \pm 20.08$ & $45.24 \pm 19.66$ & -2.840 & 0.007 \\
Duration of CIT (d) & $11.52 \pm 4.33$ & $8.20 \pm+2.77$ & 3.229 & 0.002 \\
Recovery time of CIT (d) & $19.40 \pm 3.89$ & $13.44 \pm 3.02$ & 6.051 & $<0.001$ \\
Platelet Transfusion (times) & $16(16.00)$ & $4(4.00)$ & 4.500 & 0.034 \\
rhIL-11 administered (days) & $10.68 \pm 2.46$ & $6.28 \pm 1.77$ & 7.258 & $<0.001$ \\
Bleeding (number) & $8(8.00)$ & $4(4.00)$ & 0.758 & 0.384 \\
During of bleeding (day) & $5.00 \pm 0.82$ & $4.50 \pm 0.71$ & 0.730 & 0.506 \\
\hline
\end{tabular}

$\mathrm{TP}=$ treatment phase; $\mathrm{PTP}=$ preventive treatment phase

Table 3. Toxicities between groups in TP and PTP

\begin{tabular}{lrrrr}
\hline & TP & PTP & \multicolumn{1}{c}{$\mathrm{x}^{2}$} & $p$ value \\
\hline Fever & 12 & 10 & 0.117 & 0.733 \\
Edema & 8 & 6 & 0.166 & 0.684 \\
Tachycardia & 12 & 10 & 0.177 & 0.733 \\
Muscle pain & 8 & 10 & 0.136 & 0.713 \\
Fatigue & 6 & 6 & 0.000 & 1.000 \\
Skin rash & 6 & 4 & 0.222 & 0.637 \\
Headache and dizziness & 10 & 8 & 0.136 & 0.713 \\
\hline
\end{tabular}

$\mathrm{TP}=$ treatment phase; $\mathrm{PTP}=$ preventive treatment phase

the grade of toxicities was determined according to The National Cancer Institute Common Terminology Criteria for Adverse Events (NCI CTCAE; version 3).

\section{Results}

Clinical characteristics of patients: 100 patients were recuited (male 54, female 46) and aged 21 to 58 (41.0 \pm 4.0 ) years. Non-small cell lung cancer was diagnosed in 20 patients and received chemotherapy that included paclitaxel/cisplatin (or carboplatin) or gemcitabine/ cisplatin. Small cell lung cancer were confirmed in 10 patients and received etoposide/cisplatin. Sixteen patients with breast cancer were treated with gemcitabine/cisplatin or gemcitabine/paclitaxel. Fourteen patients with gastric cancer were treated with TS-1/oxaliplatin. Another 14 patients with esophageal carcinoma were treated with fluorouracil/ cisplatin or paclitaxel/cisplatin. Ten patients with pancreatic cancer were treated with gemcitabine/ oxaliplatin. Cervical cancer was diagnosed in 6 patients and these patients were treated with paclitaxel/cisplatin. Six patients with gallbladder cancer were treated with gemcitabine/cisplatin. Four patients with ovarian cancer were treated with paclitaxel/carboplatin (Table 1).

Treatment efficacy: Duration and severity of thrombocytopenia between two phases were compared. Results: for patients in TP or PTP, nadir values of platelet were $(29.28 \pm 20.08) \times 10^{9} / \mathrm{L}$ and $(45.24 \pm 19.66) \times 10^{9} / \mathrm{L}$, duration of thrombocytopenia in TP and PTP was (11.52 \pm 4.33$)$ and $(8.20 \pm+2.77)$ days, recovery time was

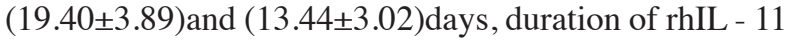
administration was $10.68 \pm 2.46)$ and $(6.28 \pm 1.77)$ days, number of patients needing platelet infusion was 16 and 4 respectively, all differences were statistically significant ( $p$ value were $0.007,0.002,0.000,0.000,0.034$ respectively). For TP and PTP, number of patients with hemorrhage was 8 and 4 , duration of bleeding was $(5.00 \pm 0.82)$ and (4.50 \pm 0.71 ) days respectively, with no statistically significant difference (Table 2).

Side effects: Adverse reactions mainly included fever, edema, arrhythmia, joint pain, fatigue, skin rash, headache, dizziness, etc., all were not statistically significant between TP and PTP (Table 3).

\section{Discussion}

Platelets are anuclear cell particles that are released into the bloodstream by megakaryocytes located in the bone marrow. The differentiation of megakaryocytes is regulated by an intricate interaction of specific cytokines and growth factors (Kaplan et al., 2006). Bone marrow stromal elements are also critical to the differentiation and release of platelets. Cancer chemotherapy often depletes the stem and progenitor cells involved in platelet proliferation, which is called CIT and leads to a diminution of platelets and temporary interruption of platelet production. CIT will be lasting until stromal elements and megakaryocytes regenerate.

Over a long period of time, platelet transfusions are the most effective methods to control bleeding and CIT (McCullough et al., 2000). However, a number of issues, eg., availability, cost, refractoriness, transfusion reactions and disease transmission related to platelet transfusion support alternate strategies to reduce or eliminate it (McCullough et al., 2000). Besides, several experimental agents targeted to prevent CIT involved in the differentiation and production of platelets (Jenkins et al., 2007). Currently, the only agent commercially available for prevention of thrombocytopenia is IL-11, but the toxicity profile seems to be concerned when it is widespread introducted into clinical practice.

In previous studies on administration of rhIL - 11 for patients with advanced breast cancer who received aggressive doses of combined chemotherapy, it was suggested a dose dependent increase in bone marrow progenitor cells, megakaryocytes, cycling megakaryocytes, and mean platelet counts, and decreased the anticipated incidence of severe CIT were associated with this administration(Orazi et al., 1996; Gordon et al., 1996). The efficacy of rHuIL-11 $(25 \mu \mathrm{g} / \mathrm{kg}$ and $50 \mu \mathrm{g} /$ $\mathrm{kg}$ ) to prevent the need for platelet transfusions in cancer patients with CIT was evaluated. The marginal activity of IL-11 to decrease the risks of thrombocytopenia was confirmed in a randomized, placebo-controlled study of rHuIL-11 to prevent CIT for patients with breast cancer and treated with dose-intensive chemotherapy (Isaacs et al., 1997). IL-11 associated toxicities reported in these studies included fatigue, anemia, myalgias, arthralgias, 
cardiovascular events, dependent edema, and fluid retention with weight gain (Gordon et al., 1996; Isaacs et al., 1997).

The efficacy of rHuIL-11 in preventing the need for platelet transfusions, compared with placebo, was invesgigated in 93 cancer patients receiving chemotherapy who had previously documented a need for platelet transfusion (Tepler et al., 1996). These patients had required platelet transfusions in the chemotherapy cycle immediately preceding entry into the study. Among the patients receiving placebo along with the chemotherapy, 96\% again experienced thrombocytopenia requiring platelet transfusion. In contrast, a $50-\mu \mathrm{g} / \mathrm{kg}$ dose of rHuIL-11 modestly reduced the platelet transfusion requirements to $70 \%(p<0.05)$ (Tepler et al., 1996). Based on these results, as well as the lack of any other available therapies besides transfusional support, the FDA approved rHuIL-11 for the treatment of CIT. Treatment-associated toxicities from the randomized study were the same as those reported in prior studies and confirmed that the use of IL-11 is frequently associated with unacceptably severe side effects. These IL-11-induced toxicities included a low incidence of atrial arrhythmias and syncope, as well as more common problems with edema and fluid retention. Despite these results with conventional chemotherapy regimens, a randomized trial of rHuIL-11 in the autologous bone marrow transplantation setting failed to demonstrate significant efficacy in decreasing platelet transfusion requirements (Vredenburgh et al., 1998). And further, few study is conducted to confirm the effectiveness of rhIL - 11 in the second prevention of CIT

In this study, our purpose is to investigate the effect and side effects of rhIL - 11 in the second prevention of CIT. We recruited Cancer patients with CIT. rhIL - 11 was administered when thrombocytopenia less than 75 $* 10^{9} / \mathrm{L}$, and in the following cycle, all these patients were treated with rhIL - 1124 hours immediately after PTP. As a result, it was suggested that for patients in TP or PTP, nadir values of platelet were $(29.28 \pm 20.08) \times 10^{9} / \mathrm{L}$ and $(45.24 \pm 19.66) \times 10^{9} / \mathrm{L}$, duration of thrombocytopenia in TP and PTP was (11.52 \pm 4.33$)$ and $(8.20 \pm+2.77)$ days, recovery time was $(19.40 \pm 3.89)$ and $(13.44 \pm 3.02)$ days, duration of rhIL - 11 administration was $10.68 \pm 2.46)$ and (6.28 \pm 1.77$)$ days, number of patients needing platelet infusion was 16 and 4 respectively, all differences were statistically significant ( $p$ value were $0.007,0.002,0.000$, $0.000,0.034$ respectively). For TP and PTP, number of patients with hemorrhage was 8 and 4 , duration of bleeding was $(5.00 \pm 0.82)$ and $(4.50 \pm 0.71)$ days respectively, with no statistically significant difference. Adverse reactions mainly included fever, edema, arrhythmia, joint pain, fatigue, skin rash, headache, dizziness, etc., all were not statistically significant between TP and PTP. In conclusion, our current study suggested that rhIL - 11 could be well tolerated and is effective that could reduce the duration, severity of thrombocytopenia, platelet transfusion, and incidence of bleeding, as well as shorten the recovery time, and duration of rhIL - 11 administration. Thus, rhIL - 11 could be commended in the second prevention of CIT for patients with cancer.

\section{Acknowledgements}

Dr. Xin-En Huang is supported by Jiangsu Provincial Special Program of Medical Science (BL2014092) and Jiangsu Province fourth stage "333 high- level Personnel Training Project" third levels of talent cultivating object.

\section{References}

Cao J, Huang XE, Liu J, et al (2014). Comparison of efficacy and toxicity of first line chemotherapy with or without epirubicin for patients with advanced stage soft tissue sarcoma. Asian Pac J Cancer Prev, 14, 7171-7.

Chen YS, Xu SX, Ding YB, et al (2014). Colorectal cancer screening in high-risk populations: a survey of cognition among medical professionals in Jiangsu, China. Asian Pac $J$ Cancer Prev, 14, 6487-91.

Chen YS, Xu SX, Ding YB, et al (2014). Helicobacter pylori Infection and the risk of colorectal adenoma and adenocarcinoma: an updated meta-analysis of different testing methods. Asian Pac J Cancer Prev, 14, 7613-9.

Clarke K, Basser RL (1996). The role of platelet growth factors in cancer therapy. Stem Cells, 14 (suppl 1), 274-280.

Cui L, Liu XX, Jiang Y, et al (2014). Phase II study on dose escalating schedule of paclitaxel concurrent with radiotherapy in treating patients with locally advanced non-small cell lung cancer. Asian Pac J Cancer Prev, 15, 1699-702.

Cui L, Sun YH, Chen J, et al (2015). Analysis of Prethrombotic States in Patients with Malignant Tumors. Asian Pac J Cancer Prev, 16, 5477-82.

Demetri GD (2000). Pharmacologic treatment options in patients with thrombocytopenia. Semin Hematol, 37 (suppl 4), 11-18.

Elting LS, Rubenstein EB, Martin CG, et al (2001). . Incidence, cost, and outcomes of bleeding and chemotherapy dose modification among solid tumor patients with chemotherapyinduced thrombocytopenia. J Clin Oncol, 19, 1137-1146.

Gordon MS, McCaskill-Stevens WJ, Battiato LA et al (1996). A phase I trial of recombinant human interleukin-11 (Neumega rHuIL-11 growth factor) in women with breast cancer receiving chemotherapy. Blood, 87, 3615-3624.

Huang XE, Cao J, Qian ZY, et al (2014). Leucogen tablets at $60 \mathrm{mg}$ three times per day are safe and effective to control febrile neutropenia. Asian Pac J Cancer Prev, 15, 8495-7.

Huang XE, Tian GY, Cao J, et al (2014). Pemetrexed as a component of first-, second- and third- line chemotherapy in treating patients with metastatic lung adenocarcinoma. Asian Pac J Cancer Prev, 14, 6663-7.

Huang XE, Wang L, Ji ZQ, et al (2015). Safety of Lienal Polypeptide Injection Combined with Chemotherapy in Treating Patients with Advanced Cancer. Asian Pac J Cancer Prev, 16, 7837-41.

Huang XE, Yan XC, Wang L, et al (2015). Thalidomide Combined with Chemotherapy in Treating Patients with Advanced Colorectal Cancer. Asian Pac J Cancer Prev, 16, 7867-9.

Isaacs C, Robert NJ, Bailey FA et al (1997). Randomized placebocontrolled study of recombinant human interleukin-11 to prevent chemotherapy-induced thrombocytopenia in patients with breast cancer receiving dose-intensive cyclophosphamide and doxorubicin. J Clin Oncol, 15, 3368-77.

Jenkins JM, Williams D, Deng Y, et al (2007). . Phase 1 clinical study of eltrombopag, an oral, nonpeptide thrombopoietin receptor agonist. Blood, 109, 4739-41.

Ji ZQ, Huang XE, Wu XY, et al (2014). Safety of Brucea javanica 
and cantharidin combined with chemotherapy for treatment of NSCLC patients. Asian Pac J Cancer Prev, 15, 8603-5.

Kaplan RN, Psaila B, Lyden D (2007). Niche-to-niche migration of bone-marrow-derived cells. Trends $\mathrm{Mol} \mathrm{Med,13,72-81.}$

Li Y, Huang XE (2015). A Pooled Analysis on Crizotinib in Treating Chinese Patients with EML4-ALK Positive Nonsmall-cell Lung Cancer. Asian Pac J Cancer Prev, 16, 4797-800.

Liu J, Huang XE (2015). Clinical application of serum tumor abnormal protein from patients with gastric cancer. Asian Pac J Cancer Prev, 16, 4041-4.

Liu J, Huang XE 1 (2014). Efficacy of Bifidobacterium tetragenous viable bacteria tablets for cancer patients with functional constipation. Asian Pac J Cancer Prev, 15, 10241-4.

Liu J, Huang XE, Feng JF (2014). Further study on pemetrexed based chemotherapy in treating patients with advanced gastric cancer (AGC). Asian Pac J Cancer Prev, 15, 6587-90.

Liu MY, Huang XE (2015). Effects of analgecine on oxaliplatininduced neurotoxicity in patients with gastrointestinal cancer. Asian Pac J Cancer Prev, 16, 4465-8.

Lu YY, Huang XE, Wu XY, et al (2014). Clinical observations on associations between the UGT1A1 genotype and severe toxicity of irinotecan. Asian Pac J Cancer Prev, 15, 3335-41.

McCullough J (2000). Current issues with platelet transfusion in patients with cancer. Semin Hematol, 37 (suppl 4), 3-10.

Orazi A, Cooper RJ, Tong J et al (1996). Effects of recombinant human interleukin-11 (Neumega rHuIL-11 growth factor) on megakaryocytopoiesis in human bone marrow. Exp Hematol, 24, 1289-97.

Qian T, Huang XE (2015). Study of Pemetrexed-based Chemotherapy for Patients with Locally Advanced or Metastatic Cancers. Asian Pac J Cancer Prev, 16, 4791-5.

Qian YD, Xu X, Wang L, Huang XE (2014). Clinical safety of chemotherapy for elderly cancer patients complicated with hypertension. Asian Pac J Cancer Prev, 15, 9875-7.

Shen K, Huang XE (2015). Clinical investigation in effect of riboflavin sodium phosphate on prevention and treatment for patients with radiotherapy related esophagitis. Asian Pac J Cancer Prev, 16, 1525-7.

Shi B, Zhang XB, Xu J, et al (2015). Systematic Analysis of Icotinib Treatment for Patients with Non-Small Cell Lung Cancer. Asian Pac J Cancer Prev, 16, 5521-4.

Sun YH, Cui L, Chen J, et al (2015). Analysis of Relationships Between Prethrombotic States and Cervical Cancer. Asian Pac J Cancer Prev, 16, 6163-6.

Tepler I, Elias L, Smith JW II et al (1996). A randomized placebocontrolled trial of recombinant human interleukin-11 in cancer patients with severe thrombocytopenia due to chemotherapy. Blood, 87, 3607-3614.

Teramura M, Kobayashi S, Yoshinaga K et al (1996). Effect of interleukin 11 on normal and pathological thrombopoiesis. Cancer Chemother Pharmacol, 38 (suppl), S99-102.

Tian GY, Miu M, Huang XE (2014). Systematic analysis of pemetrexed-based chemoradiotherapy for patients with locally advanced or metastatic esophageal cancer. Asian Pac J Cancer Prev, 15, 8475-8.

Vredenburgh JJ, Hussein A, Fisher D et al (1998). A randomized trial of recombinant human interleukin-11 following autologous bone marrow transplantation with peripheral blood progenitor cell support in patients with breast cancer. Biol Blood Marrow Transplant, 4, 134-41.

Wang L, Huang XE (2015). Clinical study on safety and efficacy of JiSaiXin (recombinant human granulocyte colony stimulating factor injection manufactured in China) for Chinese undergoing chemotherapy. Asian Pac J Cancer Prev, 16, 299-301.
Wang L, Huang XE, Cao J (2014). Clinical study on safety of cantharidin sodium and shenmai injection combined with chemotherapy in treating patients with breast cancer postoperatively. Asian Pac J Cancer Prev, 15, 5597-600.

Wu XY, Huang XE (2015). Clinical Application of Serum Tumor Abnormal Protein (TAP) in Colorectal Cancer Patients. Asian Pac J Cancer Prev, 16, 3425-8.

Wu XY, Huang XE (2015). Screening for patients with non-small cell lung cancer who could survive long term chemotherapy. Asian Pac J Cancer Prev, 16, 647-52.

Wu XY, Huang XE, Cao J, et al (2014). A predictive model for evaluating responsiveness to pemetrexed treatment in patients with advanced colorectal cancer. Asian Pac J Cancer Prev, 15, 5941-4.

Xiao Y, Liu J, Huang XE, et al (2014). Clinical study on fluvoxamine combined with oxycodone prolonged-release tablets in treating patients with moderate to severe cancer pain. Asian Pac J Cancer Prev, 15, 10445-9.

Xiao Y, Liu J, Liu YC, et al (2014). Phase II study on EANI combined with hydrochloride palonosetron for prevention of chemotherapy-induced nausea and vomiting following highly emetogenic chemotherapy. Asian Pac J Cancer Prev, 15, 3951-4.

Xu C, Huang XE, Lv PH, et al (2015). Radiofrequency Ablation in Treating Colorectal Cancer Patients with Liver Metastases. Asian Pac J Cancer Prev, 16, 8559-61.

Xu C, Huang XE, Wang SX, et al (2014). Drainage alone or combined with anti-tumor therapy for treatment of obstructive jaundice caused by recurrence and metastasis after primary tumor resection. Asian Pac J Cancer Prev, $15,2681-4$

Xu C, Huang XE, Wang SX, et al (2015). Comparison of infection between internal-external and external percutaneous transhepatic biliary drainage in treating patients with malignant obstructive jaundice. Asian Pac J Cancer Prev, 16, 2543-6.

Xu C, Lv PH, Huang XE, et al (2014). Safety and efficacy of sequential transcatheter arterial chemoembolization and portal vein embolization prior to major hepatectomy for patients with HCC. Asian Pac J Cancer Prev, 15, 703-6.

Xu C, Lv PH, Huang XE, et al (2014). Analysis of different ways of drainage for obstructive jaundice caused by hilar cholangiocarcinoma. Asian Pac J Cancer Prev, 15, 5617-20.

$\mathrm{Xu} C$, Lv PH, Huang XE, et al (2014). Internal-external percutaneous transhepatic biliary drainage for patients with malignant obstructive jaundice. Asian Pac J Cancer Prev, 15, 9391-4.

Xu C, Lv PH, Huang XE, et al (2015). Efficacy of Transarterial Chemoembolization Combined with Radiofrequency Ablation in Treatment of Hepatocellular Carcinoma. Asian Pac J Cancer Prev, 16, 6159-62.

Xu C, Lv PH, Huang XE, et al (2015). Radiofrequency Ablation for Liver Metastases after Transarterial Chemoembolization: A Systemic Analysis. Asian Pac J Cancer Prev, 16, 5101-6.

Yang L, Sun Y, Huang XE, et al (2015). Carcinoma microsatellite instability status as a predictor of benefit from fluorouracilbased adjuvant chemotherapy for stage II rectal cancer. Asian Pac J Cancer Prev, 16, 1545-51.

Zhou SB, Liu YC, Yin XX, et al (2015). Clinical observation of three dimensional conformal radiotherapy with tamoxifen in treatment of postoperative malignant glioma. Asian Pac J Cancer Prev, 16, 1743-5. 